Large arrays of chemo-mechanical nanoswitches for ultralow-power hydrogen sensing

This article has been downloaded from IOPscience. Please scroll down to see the full text article.

2010 J. Micromech. Microeng. 20105019

(http://iopscience.iop.org/0960-1317/20/10/105019)

View the table of contents for this issue, or go to the journal homepage for more

Download details:

IP Address: 128.178.195.27

The article was downloaded on 22/09/2010 at 07:40

Please note that terms and conditions apply. 


\title{
Large arrays of chemo-mechanical nanoswitches for ultralow-power hydrogen sensing
}

\author{
T Kiefer, A Salette, L G Villanueva and J Brugger \\ Microsystems Laboratory, Station 17, École Polytéchnique Fédérale de Lausanne (EPFL), \\ Lausanne 1015, Switzerland \\ E-mail: juergen.brugger@epfl.ch
}

Received 4 June 2010, in final form 27 July 2010

Published 21 September 2010

Online at stacks.iop.org/JMM/20/105019

\begin{abstract}
A hydrogen sensor based on large arrays of nanoswitches in palladium (Pd) is presented. An individual nanoswitch is realized by a suspended $\mathrm{Pd} / \mathrm{Ti} /$ poly-Si trimorph electrode and a fixed $\mathrm{Pd} / \mathrm{Ti}$ bottom electrode, which are separated by a vertical nanoscopic gap of approximately $10 \mathrm{~nm}$ in size. In hydrogen exposure, the volume expansion of Pd results in mechanical bending of the suspended electrode and the formation of an electric contact. A multitude of nanoswitches is arranged in interconnected arrays. These enable a dependence of the sensor signal on the $\mathrm{H}_{2}$ concentration by the occurrence of percolation effects. The combined use of thin film, etching and evaporation techniques allows for the large-scale fabrication of nanoswitch arrays in arbitrary topologies by design, such as parallel linear chains or square lattices. The results of hydrogen and temperature measurements are presented and discussed. In hydrogen exposure, reversible changes in the electrical resistance of up to three orders of magnitude are obtained for $\mathrm{H}_{2}$ concentrations below $4 \%$ and a power consumption down to a few picowatts.
\end{abstract}

(Some figures in this article are in colour only in the electronic version)

\section{Introduction}

Hydrogen has raised considerable interest as a future energy carrier in its application to fuel cells as it is absolutely free of polluting emissions. However, it is highly flammable in air and difficult to contain, so sensors for leak detection and monitoring are required. As a sensitive material, palladium (Pd) is the most prominent material by far due to its highly catalytic surface and large hydrogen solubility [1]. Common sensors rely on changes in resistivity upon hydrogenation [2-4], charge separation at $\mathrm{Pd}-\mathrm{SiO}_{2}$ interfaces in MOS structures [5] or single bimorph cantilevers with capacitive or optical readout $[6,7]$. They mostly require active heating elements, which lead to high power consumption. Response times are typically as long as several tens of seconds. Sensors based on nanoscopic discontinuities (nanogaps) in palladium showed improved performances with response times below $1 \mathrm{~s}$ and the possibility to operate at room temperature [8, 9]. Herein, the reversible volume expansion in palladium which occurs upon hydrogenation is used to mechanically close and reopen nanogaps, allowing an electric current to be switched across.

A single nanogap ideally behaves like a binary switch for electric current [10], but electrical transport through arrays (or networks) of nanogaps can be governed by percolation processes [11, 12]. In a typical percolation system, the nanoswitches can be replaced by electrical conductors that are randomly distributed on the sites of a two-dimensional lattice following a binary distribution. A conductor thus represents a closed nanoswitch which transports current. If such a system is placed between two electrodes, it can also be referred to as a random resistor network [11]. At a low fraction of conductors forming the network, no conductive path exists between the electrodes. Above a critical fraction, the so-called percolation threshold, a conductive cluster of electrically connected nanoswitches, eventually appears and further grows when the fraction of conductors is increased. 
A number of devices successfully employed this effect, e.g. by using break junctions in electrodeposited mesowires [8], ultrathin discontinuous palladium films [12, 13], porous silicon [14] or anodized alumina templates [15], which were mostly realized by bottom-up fabrication approaches. The major drawback of these methods is the lack of control of number and the topological arrangement of the nanogaps. Though Morris [13] pointed out that such control by design could improve the reproducibility and would be highly advantageous for industrial applications.

For the fabrication of nanogaps, a variety of techniques have been reported such as focused ion beam milling $[10,16]$, electron-beam lithography [17], nanoimprint lithography [18], AFM lithography [19], electromigration [20, 21], electrodeposition [22, 23], break junctions [8], discontinuous metal films [12] or shadow evaporation [24, 25]. Comprehensive overviews on further existing methods for nanogap fabrication can be found in the recent reviews of Li et al [26] and Prokopuk et al [27].

However, in many cases, their applicability to large arrays is limited by constraints in topological and geometrical control, lack of reproducibility [28], serial processing [10, 17] or the need for feedback-assisted techniques [23]. Further, the nanogaps are laterally defined in most cases. Suitable fabrication techniques require the highest spatial resolution which is difficult to obtain.

To this end, we developed a novel sensor concept based on large arrays of vertically defined, approximately $10 \mathrm{~nm}$ wide nanogaps which act as chemically actuated mechanical nanoswitches. Geometry and topology of the arrays can be controlled by the sole use of standard microfabrication techniques. Basically, the device combines three generic mechanisms: (i) the deflection of a poly-Si/Ti/Pd trimorph electrode upon hydrogen exposure, (ii) the reversible closing of a nanoswitch and (iii) percolation in an interconnected network. A thin silicon dioxide $\left(\mathrm{SiO}_{2}\right)$ film is used as a spacer to define the size of the nanogaps in the vertical direction which facilitates the parallel fabrication of a multitude of nanoscopic features on a large scale. Shadow evaporation across a vertical edge as a central process step allows for the creation of the trimorph electrode and the nanogaps at the same time. We realized a single microwire that is interrupted by two nanogaps and two different network topologies: parallel linear chains, inspired by the result of electrochemically deposited mesowires on highly ordered pyrolytic graphite (HOPG) surfaces [8, 9], and a square lattice structure which commonly serves as a model system for percolation processes.

\section{Principle and design}

\subsection{Response of palladium to hydrogen exposure}

The two major effects that are observed upon hydrogen absorption in $\mathrm{Pd}$ are a reversible change in resistivity and in volume, respectively [1]. Dissolved $\mathrm{H}$ atoms occupy interstitial octahedral sites within the Pd lattice which leads to an increased scattering of conducting electrons. This in turn increases the resistivity of the material. The volume expansion of $\mathrm{Pd}$ is a result of a phase transformation in hydrogen contents above $\mathrm{H} / \mathrm{Pd}=0.01$ (the so-called $\beta$-phase) where the lattice constant increases from $3.890 \AA$ to $4.025 \AA$. Below, in the hydrogen poor $\alpha$-phase, the lattice expands to $3.895 \AA$ for bulk Pd [29]. For nanocrystalline Pd such as nanoclusters or evaporated/sputtered thin films, the phase transformation has found to be shifted up to hydrogen contents between 0.03 and $0.1 \mathrm{H} / \mathrm{Pd}$, caused by an increased hydrogen solubility in grain boundaries [30, 31]. Further, the effective material expansion in the hydrogen poor $\alpha$-phase is larger as compared to bulk materials [32], which makes nanocrystalline palladium advantageous in nanogap-based sensing applications.

\subsection{Operation principle of a single nanoswitch}

A single, elementary sensing element within the array consists of a single-side clamped, suspended trimorph poly-Si/Ti/Pd electrode which is separated from a second $\mathrm{Ti} / \mathrm{Pd}$ electrode by a vertical spacer, leaving a nanoscopic vertical gap as illustrated in figure 1(a). Upon hydrogenation, the palladium lattice expands and induces a compressive stress in the Pd film. This leads to a deflection of the trimorph along its vertical axis. The titanium layer (the thin intermediate line in figure 1(a)) improves the adhesion between poly-Si and Pd in order to avoid delamination of the Pd film during hydrogenation [10]. If the deflection is large enough, the hydrogenated metal connects to its counterpart at the bottom and forms an electric contact (figure 1(b)). Upon hydrogen desorption, the original lattice constant is restored, resulting in mechanical relaxation of the trimorph and the interruption of the electrical connection from the bottom metal electrode.

\subsection{Sensor design}

A multitude of such elementary nanoswitches are arranged into interconnected arrays. Hereby, we exploit the fact that the size of the nanogaps is subject to smallest variations. These variations are a result of local fluctuations on the metal surface, caused by the granular metallic microstructure. The width of the statistical gap-size distribution gives rise to electrical transport by percolation with a dependence on the $\mathrm{H}_{2}$ concentration. The topological structure of the nanoswitch arrays is defined by creating a network of $\mathrm{Ti} / \mathrm{Pd}$ microwires that are placed on top of an array of ring-shaped suspended poly-Si structures (in the following also referred to as ring membranes). The edges of the suspended poly-Si ring membranes define the locations where the microwires are interrupted by nanogaps. The topology is given by the number of adjacent nanoswitches that can form a conductive pathway in a closed state, and is expressed by the coordination number (number of next neighbors) $z$. For arrays of microwires that form a square-shaped lattice, a coordination number of 4 is obtained (in the following referred to as z4-devices). Microwires arranged in parallel result in a coordination number of 2 (therefore referred to as z2-devices). Placed in between two electrodes, electrical conduction across the network occurs as soon as the critical number of elementary connections is reached (figure $1(c)$ ). By further increasing the 

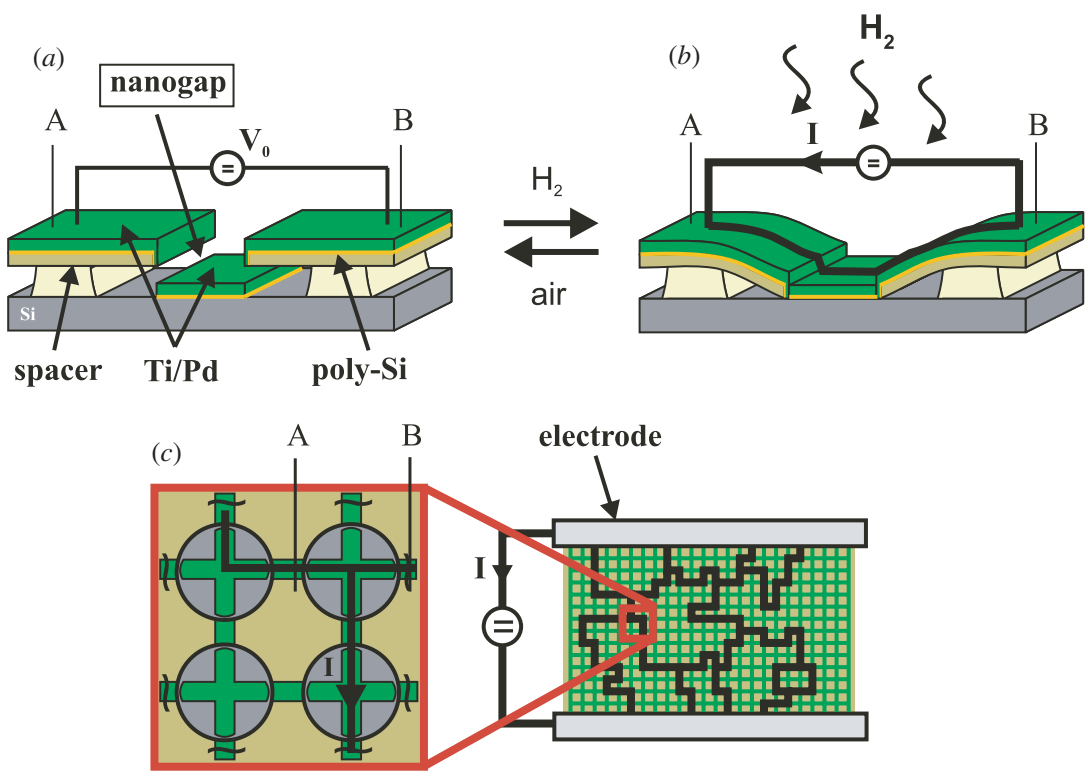

Figure 1. Schematic representation of the operating principle, based on an interconnected array of elementary trimorph poly-Si/Ti/Pd nanoswitches. (a) Cross-sectional view of two adjacent switches, consisting of a palladium (Pd) microwire which is situated on top of a short-suspended poly-Si structure and a bottom Ti/Pd electrode which is $\approx 10 \mathrm{~nm}$ apart. In a non-sensing state, the elementary switches remain open and prevent an electric current to flow. $(b)$ Upon hydrogenation, Pd expands and deflects the trimorph. An ohmic contact with the bottom electrode is formed and an electric current $I$ can flow. $(c)$ Electrically interconnected elementary switches are arranged into a percolation network; here the top view of the interconnected network of wires and nanogaps. A sufficient number of closed nanogaps form a conductive path between external electrodes. This process is reversible.

number of connections, more conductive paths form and lead to an increase in the overall current.

The optimum thicknesses for the thin films were estimated using finite element analysis (FEA). The trimorph electrodes were chosen to be as short as $500 \mathrm{~nm}$, motivated by the need for a sufficient rigidity to avoid mechanical fluctuations which could be expected from a low elastic constant. The spring constant $k$ of the electrodes is around $300 \mathrm{~N} \mathrm{~m}^{-1}$, resulting in a resonance frequency $f_{\text {res }}$ of around $100 \mathrm{MHz}$. The thickness of the spacer between substrate and poly-Si was set to $90 \mathrm{~nm}$, and the thickness of poly-Si to $50 \mathrm{~nm}$. Therewith, an optimum thickness of $15 \mathrm{~nm}$ and $73 \mathrm{~nm}$ for titanium and palladium, respectively, was calculated for maximal deflection. This results in a nanoscopic gap in the order of some nanometers.

\section{Fabrication}

Simple and scalable top-down microfabrication techniques are used to fabricate the devices. The nanogaps and the trimorph electrodes are created by shadow evaporation of microwires across the freestanding poly-Si ring membrane in the same step, which allows for the fabrication of a multitude of nanoscale features on a large scale.

The process flow is schematically shown in figure 2 . It starts with the uniform deposition of two thin films on a standard $100 \mathrm{~mm}$ silicon wafer: a $90 \mathrm{~nm}$ thin thermally grown $\mathrm{SiO}_{2}$ and a $50 \mathrm{~nm}$ thin undoped poly-Si film that is deposited by low-pressure chemical vapor deposition (LPCVD) (see figure 2(a)). The $\mathrm{SiO}_{2}$ layer will later serve as the vertical spacer that defines the distance between the trimorph and the bottom metal electrode. Standard deviations for the thickness of the spacer were obtained by spectro-reflectometry and were around $1 \mathrm{~nm} \pm 0.3 \mathrm{~nm}$ across the wafer. The role of the poly-Si film is to define a first array pattern consisting of circular openings with a diameter of $12 \mu \mathrm{m}$, which is used to create the nanogaps. In a first photolithographic step, the array of circular openings is created in the poly-Si film using ion-coupled plasma (ICP) dry etching and $\mathrm{SF}_{6} / \mathrm{C}_{4} \mathrm{~F}_{8}$ as an etching gas (figure $2(b)$ ). This pattern is transferred into $\mathrm{SiO}_{2}$ by wet etching using buffered hydrofluoric acid (BHF). By overetching, an undercut of the poly-Si is obtained. The resulting freestanding structure eventually serves as the suspended ring membrane on which the metal wires are deposited (figure 2(c)). In a second photolithographic step, a wire pattern is created which connects the openings according to the desired topology of the network. The width of each wire is $3.5 \mu \mathrm{m}$. The two metal layers ( $15 \mathrm{~nm} \mathrm{Ti}$ and $73 \mathrm{~nm} \mathrm{Pd})$ are then evaporated by electron-beam evaporation. The shadow effect in the vertical edge of the underetched poly-Si ring membrane interrupts the deposited metallic wires and leaves a nanoscopic gap (figure $2(d)$ ). Scanning electron microscope (SEM) images showed that after removal of the photoresist and the excessive metal in a lift-off bath, the membranes remain straight and no stiction or significant bending caused by internal stresses of the multilayer structures occurs. The gap size was estimated by SEM analysis to be around $10 \mathrm{~nm}$. Four point probe measurements on a blank wafer that was entirely covered with $\mathrm{Pd}$ were used to determine the homogeneity of the film thickness across the wafer. Using the relation $t=\rho / R_{\mathrm{S}}$, where $\rho$ is the resistivity of the material and $R_{\mathrm{S}}$ is the measured square resistance, a standard deviation of $\sigma \approx 1.5 \mathrm{~nm}$ was obtained. The mean grain size was estimated 
(a)

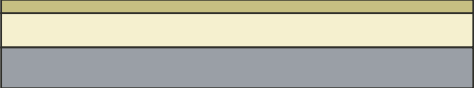

(b)

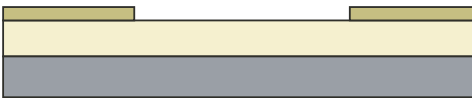

(c)

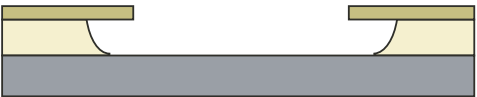

(d)

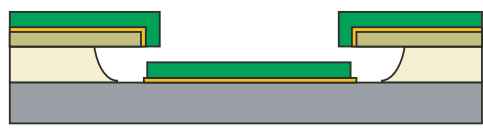

(e)

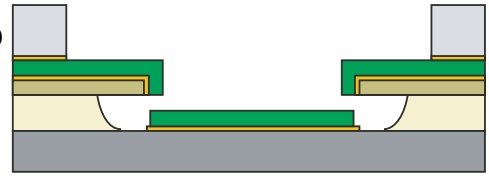

$\mathrm{Si}$

p-Si :
Pd :

Al :

Figure 2. Schematic process flow for sensor fabrication: $(a)-(c)$ creation of a suspended poly-Si ring membrane by dry and wet etching, $(d)$ the trimorph and nanogaps are created by shadow evaporation of Ti/Pd microwires across the vertical poly-Si edge, $(e)$ deposition of electrodes.

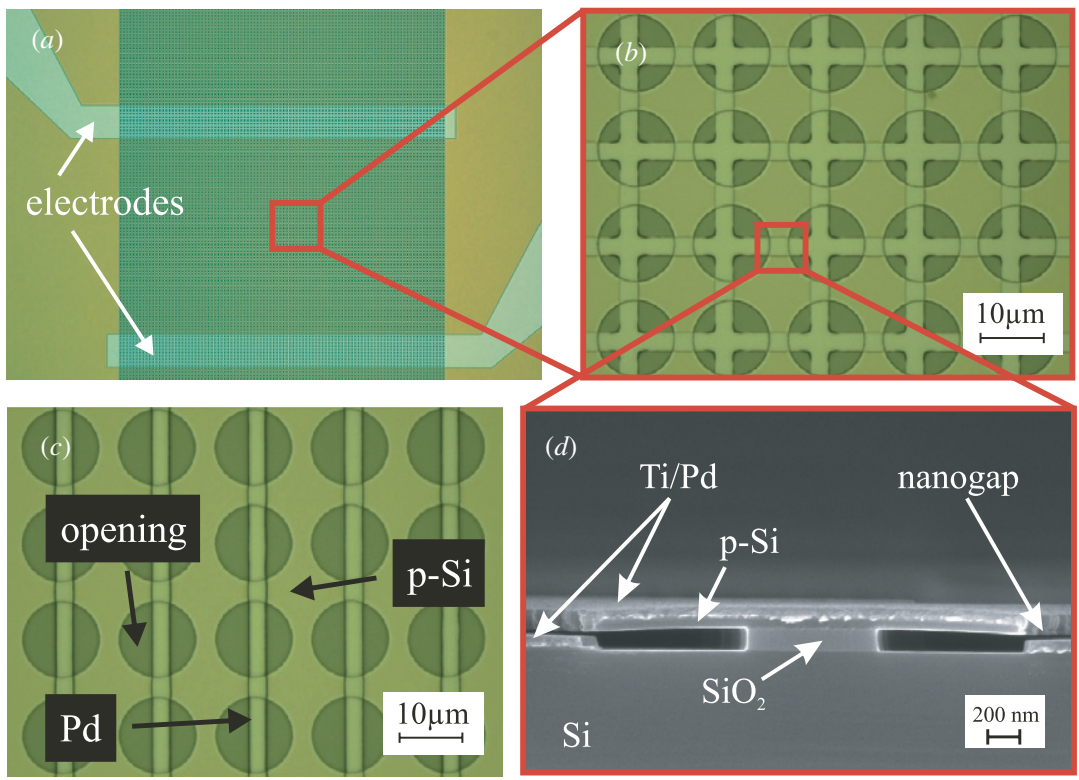

Figure 3. Optical images of nanogap networks with different coordination numbers: $(a)$ entire sensing area with electrodes, $(b)$ network with $z=4,(c)$ network with $z=2,(d)$ cross-sectional SEM image of two adjacent nanogaps.

using XRD analysis. Measuring the broadened width of the $(1,1,1)$ diffraction peak [33] yielded an average grain size of approximately $19 \mathrm{~nm}$. It corresponds to estimates from SEM image analysis. Aluminum (Al) electrodes and contact pads for wire bonding, including a thin $\mathrm{Ti}$ adhesion layer, are deposited in a final photolithographic, evaporation and liftoff step. The distance between the electrodes is $900 \mu \mathrm{m}$, resulting in a total number of 120 nanoswitches in this direction. In the horizontal direction, 100 wires are arranged in parallel, resulting in 198 nanoswitches in that direction for the square lattice. Figure $3(a)$ shows an optical image of a sensing area and figure $3(b)$ a closeup of the containing square topology (z4-device). In figure 3(c) a respective z2-device comprising parallel linear chains of nanogaps is displayed. A cross-sectional SEM image of two adjacent nanoswitches is presented in figure $3(d)$, showing the suspended trimorph electrodes that are anchored to the $\mathrm{SiO}_{2}$ spacer. The final chip was $7.6 \mathrm{~mm} \times 7.6 \mathrm{~mm}$ in size.
Further, reference devices consisting of continuous palladium microwire networks were fabricated. In this case, the same process as described above is used except that the etching of both the poly-Si and the $\mathrm{SiO}_{2}$, which create the circular openings, is omitted. Consequently, the microwires are electrically continuous and do not exhibit any mechanical degrees of freedom.

\section{Experimental results}

\subsection{Characterization methods}

The sensors were wire-bonded into standard dual-in-line (DIL) ceramic packages (Spectrum Semiconductor Materials, Inc., CERDIP 28-DIL) and mounted onto a printed circuit board (PCB). The PCB, which provides an external electrical connection, was placed into a sealed flow cell (out of poly(methyl-methacrylate), PMMA) for hydrogen 

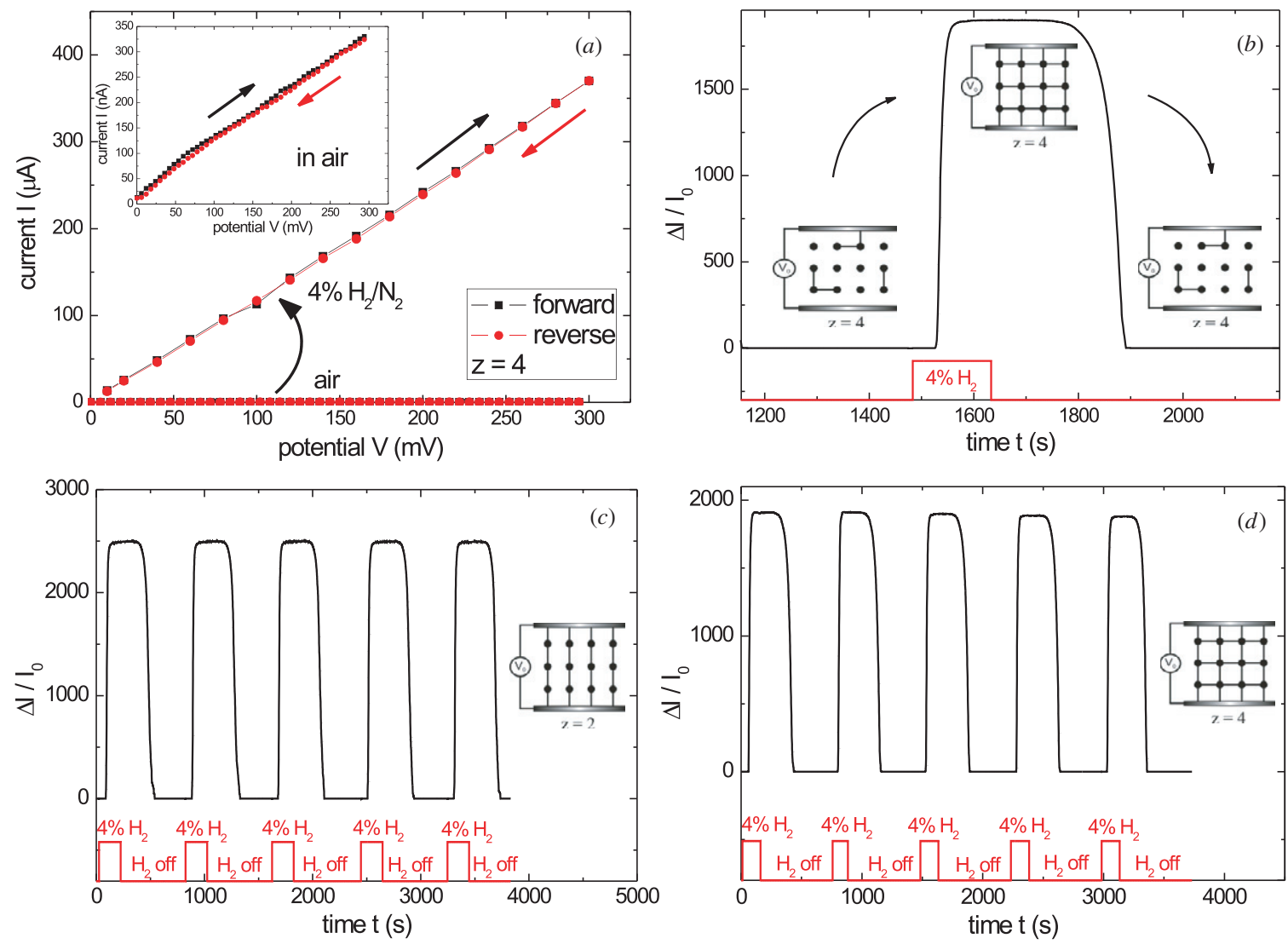

Figure 4. (a) Saturation current in air and in a hydrogen atmosphere $\left(4 \% \mathrm{H}_{2} / \mathrm{N}_{2}\right)$ as a function of the applied bias potential. The linearity under $\mathrm{H}_{2}$ and the low resistances indicate ohmic conduction. (b) Typical reversible sensor response to $4 \% \mathrm{H}_{2} / \mathrm{N}_{2}$. (c), (d) Sensor response to periodic hydrogen $\left(4 \% \mathrm{H}_{2} / \mathrm{N}_{2}\right)$ /air cycles for a z2- and z4-device, respectively. Applied electrical potential: $100 \mathrm{mV}$. A stable saturation and initial baseline values are reached at each cycle. Relative changes in resistance of more than three orders of magnitude are obtained.

measurements. Two gas lines, a $4 \% \mathrm{H}_{2} / \mathrm{N}_{2}$ gas pre-mixture (99.999\%) and pure $\mathrm{N}_{2}$ gas (99.999\%), were used and mixed before the cell at a constant flow rate of $200 \mathrm{ml} \mathrm{min}^{-1}$, if needed. The gas flow rates were controlled by electronic mass flow controllers (Vögtlin Instruments AG). The outlet gas line was short as compared to the inlet gas lines in order to avoid excessive gas pressures inside the chamber. The sensors were biased with an electric dc potential of $100 \mathrm{mV}$ by the use of an Agilent 34410A Digit Multimeter. In order to exclude any influences caused by the experimental procedure, the sensors were tested toward various flow rates between $25 \mathrm{ml} \mathrm{min}^{-1}$ and $200 \mathrm{ml} \mathrm{min}^{-1}$ for a $4 \% \mathrm{H}_{2} / \mathrm{N}_{2}$ gas mixture. No change in current was observed in the considered range of flow rates. Further, the sensor response to pure $\mathrm{N}_{2}$ gas was tested and showed no significant change in current as compared to the baseline in air.

Prior to the measurements, the devices were exposed to a $4 \% \mathrm{H}_{2} / \mathrm{N}_{2}$ gas mixture for $90 \mathrm{~min}$ and recovered for at least $15 \mathrm{~min}$ in air for priming. Electrical measurements with the reference devices showed that this recovery time is sufficient to fully restore pure Pd from its hydrogenated state.

\subsection{Sensor characteristics in hydrogen}

The reference devices showed ohmic electrical behavior and the expected instantaneous increase in resistance upon hydrogen exposure. The electrical resistances of single $400 \mu \mathrm{m}$ long wires and both network topologies were about $250 \Omega$ and $4 \Omega$, respectively, which agree with the theoretical values that are $245 \Omega$ and $2.5 \Omega$, respectively. Note that the nanocrystalline structure of the evaporated films results in an increased electrical resistivity as compared to bulk material [34], and was around $19 \times 10^{-8} \Omega \mathrm{m}$.

The sensing devices including nanogaps exhibited electrical resistances of some $100 \mathrm{k} \Omega$ up to $1 \mathrm{M} \Omega$ in air. The $I-V$ characteristics showed linear behavior for low voltages (see the inset in figure 4(a)). Upon hydrogen exposure, a strong increase in current (decrease in resistance) of up to several orders of magnitude was observed. This change in resistance is extremely high as compared to other sensors $[8,14,35-37]$ and is related to the fact that a controlled assembly of mechanical switches is used to create electrical contacts. Resistances in the hydrogenated state were around $250 \Omega$ for the $\mathrm{z} 2$-devices and around $310 \Omega$ for the $\mathrm{z} 4$-devices. In figure $4(a)$, the saturation current during a $4 \%$ hydrogen exposure as a function of the applied bias potential for a $\mathrm{z} 4$ device shows linear behavior as it is expected from an ohmic conductor. Together with the extremely low values for the resistance it confirms the existence of ohmic contacts between the metallic counterparts of the nanoswitches. Further, no hysteresis effects were observed in the considered bias range 
(a)

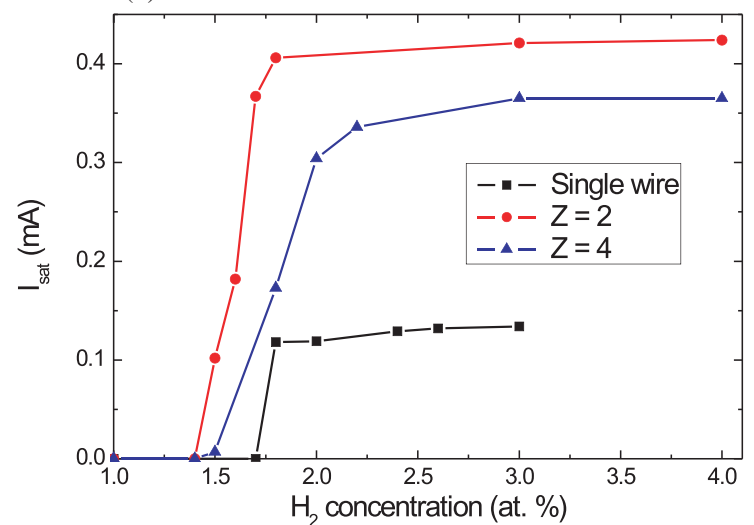

(c) $\mathrm{Z}=2$

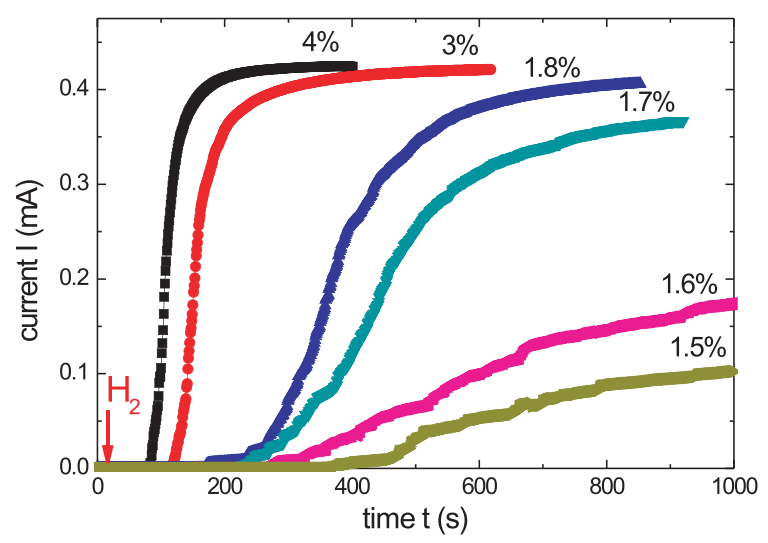

(b) single wire

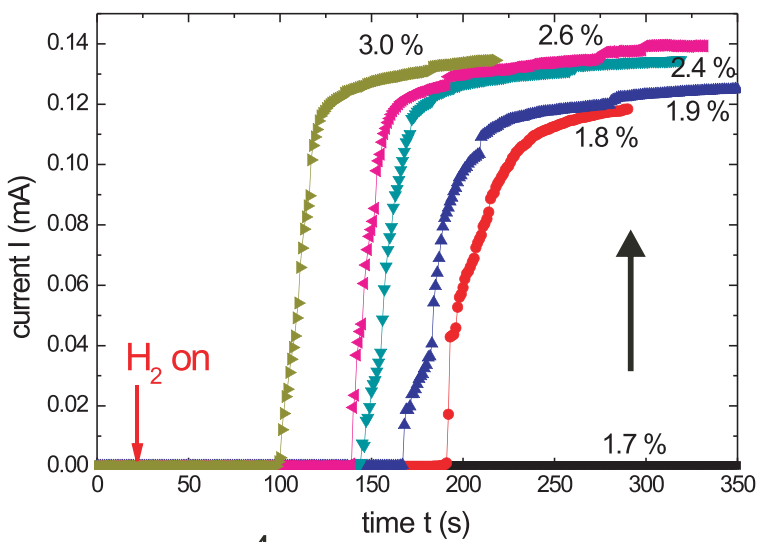

(d) $\mathrm{Z}=4$

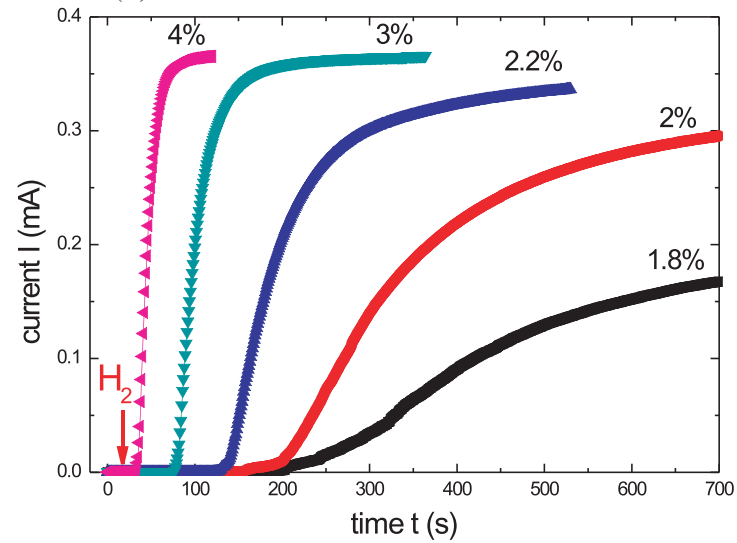

Figure 5. (a) Dependence of sensors with different topologies to various hydrogen concentrations. The sensing range is narrow and between $1.5 \%$ and $3 \% \mathrm{H}_{2} / \mathrm{N}_{2}$ and switch-like for the single-wire device. $(b)-(d)$ Typical temporal responses for different devices. For the single-wire device, mainly a change in delay time and little change in the saturation current are observed. For z 2 and $\mathrm{z} 4$ devices, the saturation current and the slope of the response signal decrease strongly for lower hydrogen concentrations.

of 0-300 $\mathrm{mV}$ in air or hydrogen. A typical response to a $4 \%$ $\mathrm{H}_{2} / \mathrm{N}_{2}$ gas mixture is shown in figure $4(b)$. It presents the relative change in the current $\Delta I / I_{0}$, where $I_{0}$ is the baseline current in air. After removing the gas flow, the resistance recovered back to its original value in air. Upon hydrogenation, the typical decrease in the current of a continuous palladium structure holds off, which indicates that indeed no continuous conductive path exists in air (as illustrated by the schematic in the lower-left corner of figure $4(b)$ ).

The switching mechanism is completely reversible, which is demonstrated by alternating hydrogen/air cycles (see figures $4(c)$ and $(d)$ for the two different network topologies). The sensor reversibly switches the resistance by up to more than three orders of magnitude without any significant drift of the saturation value. This allows us to drive the sensors at bias potentials even as low as $1 \mathrm{mV}$, without severely compromising the signal-to-noise ratio. The power consumption in a nonsensing state is then found to be as small as some few picowatts only. This is orders of magnitude lower than the lowest power consumption reported so far which is about $1 \mathrm{nW}$ [35].

Note that preceding the steep response pulse to $\mathrm{H}_{2}$, a delay time of some seconds is observed (figure $4(b)$ ). It is associated with the time which is needed to bend the trimorph until a mechanical contact is created. Higher $\mathrm{H}_{2}$ concentrations resulted in shorter delay times which is due to the enhanced kinetics of the hydrogen absorption process.

The dependence of the saturation current $I_{\text {sat }}$ on the hydrogen concentration is shown in figure $5(a)$. For comparison, single wires comprising merely two gaps in series were measured, which showed a sharp transition from the non-conducting to the conducting state for increasing $\mathrm{H}_{2}$ concentrations. A typical response is shown in figure $5(b)$, where the critical concentration for conduction was between $1.7 \%$ and $1.8 \% \mathrm{H}_{2} / \mathrm{N}_{2}$. The delay time before the first reaction to $\mathrm{H}_{2}$ might vary from device to device, caused by process-related statistical spread in size of the nanogaps. In contrast, the network topologies have a dependence on the $\mathrm{H}_{2}$ concentration in a narrow range between $1.5 \%$ and $3 \%$. The fact that this dependence was only observed for networks of nanogaps leads to the conclusion that percolation transport occurs, where the electrical resistance depends on the number of conducting paths through the array. It is caused by the statistical distribution of the threshold hydrogen concentrations for nanogap conduction [38]. Below 1.5\%, no response was observed because the lattice expansion in the $\alpha$ phase was not sufficient yet to close the gaps of the given size. This detection limit could be improved by a further decrease in gap size. This would require a precise fine tuning of the thin 
film deposition conditions in order to obtain a reproducible result. In fact, many Pd nanogap sensors suffer from such a detection limit. Besides by decreasing the gap size it could also be overcome by decreasing the grain size of the Pd film to below $10 \mathrm{~nm}$, where a highly increased $\mathrm{H}_{2}$ solubility and lattice expansion in the $\alpha$-phase have been observed [36, 39]. Typical 0-90\% response times were down to $30 \mathrm{~s}$ for a $\mathrm{H}_{2}$ concentration of $4 \%$ and at least $800 \mathrm{~s}$ for $\mathrm{H}_{2}$ concentrations of about $1.5 \%$. This is slower than what has been obtained using discontinuous Pd films [36] or Pd mesowires [8, 9] which showed response times of a few seconds or less. It is however comparable with performances of MOS-based devices that might show response times of several minutes [37].

A striking feature of the network topologies compared to the single-wire devices is the change in dynamic behavior (see figures $5(b)-(d)$ ). For the single-wire devices, the delay time increases for reduced hydrogen concentrations whereas the slope of the response signal remains almost constant. Here, the slower chemical kinetics that are present at lower concentrations decelerate the mechanical response of the trimorphs. For devices containing wire networks, apart from a similar change in delay time, a very pronounced drop in slope is obtained for decreasing concentrations. We interpret this behavior as a result of statistically distributed delay times and the resulting incremental formation of conductive paths in the percolation network. Thereto, we can associate each small incremental increase in current with the formation of a new conductive path by closing of nanogaps with successively increasing delay times. For low concentrations, only a fraction of nanogaps will close. Due to the deceleration of the absorption process with time, new pathways will form and stepwise contribute to the conductivity until the process is entirely completed. For higher concentrations, where the equilibrium current reaches the saturation value of the network, most of the paths are formed at an earlier phase of the absorption process and hence occur on a faster timescale. This particular characteristic of the sensor response enables the possibility of not only using the amplitude of the response pulse for $\mathrm{H}_{2}$ detection but also its slope. By implementing the slope dependence into the signal processing unit, the response times of the sensor could be highly reduced, especially at lower hydrogen concentrations. In that case, data on the $\mathrm{H}_{2}$ concentration would become available far before an equilibrium state is reached.

It should be mentioned that our current results further give indications for more reproducible sensing characteristics of the network topologies as compared to the single wires. This finding implies that the reproducibility of a single nanoscopic element that is compromised by statistical spread of the fabrication process can be improved by arranging a multitude into interconnected networks. Such behavior is theoretically predicted by scaling theory [40] and should be the subject of future investigations.

\subsection{Temperature dependence}

In addition, the temperature dependence of the sensor resistance was studied in air. The temperature coefficient of resistance (TCR) $(1 / R) \mathrm{d} R / \mathrm{d} T$ was found to be negative and not constant in a temperature between $23{ }^{\circ} \mathrm{C}$ and $200{ }^{\circ} \mathrm{C}$. The electric current in air increased by a factor of 2 at $70{ }^{\circ} \mathrm{C}$ and by a factor of 100 at $200{ }^{\circ} \mathrm{C}$. Negative TCRs are typical for discontinuous metallic structures [41], which confirms that no conductive path exists in air. Such characteristics have been reported for instance in discontinuous ultrathin metal films and could often be explained by thermally activated quantum tunneling processes that take place between the isolated metal islands [41-43]. The activation process results in a logarithmic dependence of the electric current $I$ on the inverse temperature, $I \propto \exp (-\delta E / k T)$, which is not the case for our devices. Due to the large metallic islands and small gaps, the activation energy $\Delta E$ for thermally generated charge carriers is high and the corresponding contribution to the tunneling current negligible. Since thermal expansion of the trimorph electrode likewise results in vertical mechanical bending, we attribute a part of the increase in the current $I$ in the low temperature range $T \leqslant 70{ }^{\circ} \mathrm{C}$ to a gradual decrease in the tunneling gap, caused by thermal bending. Above, the transition beyond the percolation threshold is likely to occur. Other contributions are likely to be related to the presence of superficial water films that bridge the nanogaps. Further investigations are necessary to confirm this hypothesis and to explain the complex behavior. Nonetheless, the signal change with temperature is still orders of magnitude lower than that upon hydrogen exposure.

\section{Conclusions}

In summary, we presented a novel palladium-based hydrogen sensor using arrays of mechanical nanoswitches wherein three generic mechanisms were combined: (i) a bimorph effect, induced by the volume expansion of palladium during hydrogenation, (ii) the reversible mechanical closing and reopening of a nanogap and (iii) percolation in an interconnected array or network. The use of shadow evaporation as a key element in fabrication allowed for the creation of highly ordered nanogap arrays with a specific geometry and topology on a large scale. The devices operate at room temperature and exhibit relative changes in resistance of several orders of magnitude upon hydrogen exposure below the lower explosion limit of hydrogen in air. Thereby, an extremely low power consumption down to some picowatts can be obtained. The response is reversible and without any significant drift after several hydrogen/air cycles. Although an optimization of the response time is still desirable, this sensor concept shows high potential for mobile low-power applications, and the sole use of standard MEMS technologies makes it interesting for industrial manufacturing.

From a general point of view, this working principle could likewise be applied to any bi- or multilayer system, wherein an environmental influence induces mechanical stress in one of the layers. If the stress is compressive and sufficiently high, the nanoscopic gaps can be closed and contribute to a percolation current across the network. This opens up the possibility of realizing sensors for the detection of temperature [44] or biological species [45, 46], or to design an experimental platform to study percolation mechanisms 
under defined geometrical and topological conditions. Future work may further focus on the investigation of influences of the sensor geometry and topology as we recently showed in [38]. The present device is therefore not only an interesting candidate for future sensor developments but could also serve as an experimental platform to study nanoelectromechanical (NEMS) percolation lattices under well-controlled conditions.

\section{Acknowledgments}

The authors thank the FP6 Integrated Project HySYS SES6-019981 for financial support, the Center of MicroNanofabrication (CMI) at EPFL in Lausanne for technical support, Dr Fred Favier for helpful discussions and Dr Michel Rossi for kindly supplying equipment for the hydrogen measurement setup.

\section{References}

[1] Lewis F A 1976 The Palladium Hydrogen System (New York: Academic)

[2] Cabrera A L and Aguayo-Soto R 1997 Hydrogen absorption in palladium films sensed by change in their resistivity Catal. Lett. 45 79-83

[3] Frazier G A and Glosser R 1980 Characterization of thin films of palladium-hydrogen system J. Less-Common Met. 74 89-96

[4] Volkening F A, Naidoo M N, Candela G, Holtz R L and Provenzanoe V 1995 Characterization of nanocrystalline palladium for solid state gas sensor applications Nanostruct. Mater. 5 373-82

[5] Lundström I, Shivaraman M S and Svensson C 1977 Chemical reactions on palladium surfaces studied with Pd-MOS structures Surf. Sci. 64 497-519

[6] Baselt D R, Fruhberger B, Klaassen E, Cemalovic S, Britton C L Jr, Patel S V, Mlsna T E, McCorkle D and Warmack B 2003 Design and performance of a microcantilever-based hydrogen sensor Sensors Actuators B 88 120-31

[7] $\mathrm{Hu} Z$, Thundat $\mathrm{T}$ and Warmack R J 2001 Investigation of adsorption and absorption-induced stresses using microcantilever sensors J. Appl. Phys. 90 427-31

[8] Favier F, Walter E C, Zach M P, Benter T and Penner R M 2001 Hydrogen sensors and switches from electrodeposited mesowire arrays Science 293 2227-31

[9] Walter E C, Favier F and Penner R M 2002 Palladium mesowire arrays for fast hydrogen sensors and hydrogen-actuated switches Anal. Chem. 74 1546-53

[10] Kiefer T, Favier F, Vazquez-Mena O, Villanueva G and Brugger $\mathrm{J} 2008 \mathrm{~A}$ single nanotrench in a palladium microwire for hydrogen detection Nanotechnology 19125502

[11] Kirkpatrick S 1973 Percolation and conduction Rev. Mod. Phys. 45 574-88

[12] Dankert O and Pundt A 2002 Hydrogen induced percolation in discontinuous films Appl. Phys. Lett. 81 1618-20

[13] Morris J E 1998 Recent developments in discontinuous metal thin film devices Vacuum 50 107-13

[14] Luongo K, Sine A and Bhansali S 2005 Development of a highly sensitive porous Si-based hydrogen sensor using Pd nano-structures Sensors Actuators B 111-112 125-9

[15] Kim K T, Sim S J and Cho S M 2006 Hydrogen gas sensor using Pd nanowires electro-deposited into anodized alumina template IEEE Sensors J. 6 509-13
[16] Nagase T, Gamo K, Kubota T and Mashiko S 2006 Direct fabrication of nano-gap electrodes by focused ion beam etching Thin Solid Films 499 279-84

[17] Fabrizio E Di, Grella L, Baciocchi M, Gentilia M, Ascoli C, Cappella B, Frediani C and Morales P 1997 Nanometer biodevice fabrication by electron beam lithography $J$. Vac. Sci. Technol. B 15 2892-6

[18] Austin M D, Ge H, Wu W, Li M, Yu Z, Wasserman D, Lyon S A and Chou S Y 2004 Fabrication of $5 \mathrm{~nm}$ linewidth and $14 \mathrm{~nm}$ pitch features by nanoimprint lithography Appl. Phys. Lett. 84 5299-301

[19] Notargiacomo A, Foglietti V, Cianci E, Capellinik G, Adami M, Faraci P, Evangelistik F and Nicolini C 1999 Atomic force microscopy lithography as a nanodevice development technique Nanotechnology 10 458-63

[20] Ward D R, Grady N K, Levin C S, Halas N J, Wu Y, Nordlander P and Natelson D 2007 Electromigrated nanoscale gaps for surface-enhanced raman spectroscopy Nano Lett. 7 1396-400

[21] Mahapatro A K, Ghosh S and Janes D B 2006 Nanometer scale electrode separation (nanogap) using electromigration at room temperature IEEE Trans. Nanotech. 5 232-6

[22] Deshmukh M M, Prieto A L, Gu Q and Park H 2003 Fabrication of asymmetric electrode pairs with nanometer separation made of two distinct metals Nano Lett. 3 1383-5

[23] He H X, Boussaad S, Xu B Q, Li C Z and Tao N J 2002 Electrochemical fabrication of atomically thin metallic wires and electrodes separated with molecular-scale gaps J. Electroanal. Chem. 522 167-72

[24] Lefebvre J, Radosavljevic M and Johnsona A T 2000 Fabrication of nanometer size gaps in a metallic wire Appl. Phys. Lett. 76 3828-30

[25] Dirk S M, Howell S W, Zmunda S, Childs K, Blain M, Simonson R J and Wheeler D R 2005 Novel one-dimensional nanogap created with standard optical lithography and evaporation procedures Nanotechnology 16 1983-5

[26] Li T, Hu W and Zhu D 2010 Nanogap electrodes Adv. Mater. 22 286-300

[27] Prokopuk N and Son K-A 2008 Alligator clips to molecular dimensions J. Phys.: Condens. Matter 20374116

[28] Harkay J R and Crowell A D 1976 Measurement and analysis of temperature-dependent $\mathrm{rf}$ impedance of discontinuous metal films on glass substrates $J$. Appl. Phys. 47 4504-8

[29] Flanagan T B and Oates W A 1991 The palladium hydrogen system Annu. Rev. Mater. Sci 21 269-304

[30] Pundt A and Kirchheim R 2006 Hydrogen in metals: microstructural aspects Annu. Rev. Mater. Res. 36 555-608

[31] Kishimoto S, Inoue M, Yoshida N and Flanagan T 1986 Solution of hydrogen in thin palladium films J. Chem. Soc. Faraday Trans. 182 2157-84

[32] Lemier C and Weissmüller J 2007 Grain boundary segregation, stress and stretch: effects on hydrogen absorption in nanocrystalline palladium Acta Mater. 55 1241-54

[33] Patterson A L 1939 The Scherrer formula for X-ray particle size determination Phys. Rev. 56 978-982

[34] Reale C 1971 Properties of the conduction electrons in the metals of group VIII J. Mater. Sci. 6 33-8

[35] Offermans P, Tong H D, van Rijn C J M, Merken P, Brongersma S H and Crego-Calama M 2009 Ultralow-power hydrogen sensing with single palladium nanowires Appl. Phys. Lett. 94223110

[36] Xu T, Zach M P, Xiao Z L, Rosenmann D, Welp U, Kwok W K and Crabtree G W 2005 Self-assembled monolyer-enhanced hydrogen sensing with ultrathin palladium films Appl. Phys. Lett. 86203104

[37] Briand D, Wingbrant H, Sundgren H, van der Schoot B, Ekedahl L-G, Lundström I and de Rooij N F 2003 
Modulated operating temperature for MOSFET gas sensors: hydrogen recovery time reduction and gas discrimination Sensors Actuators B 93 276-285

[38] Kiefer T, Villanueva G and Brugger J 2009 Conduction in rectangular quasi-one-dimensional and two-dimensional random resistor networks away from the percolation threshold Phys. Rev. E 80021104

[39] Suleiman M, Jisrawi N M, Dankert O, Reetz M T, Bähtz C, Kirchheim R and Pundt A 2003 Phase transition and lattice expansion during hydrogen loading of nanometer sized palladium clusters J. Alloys Compd. 356-357 644-8

[40] Stauffer D 1979 Scaling theory of percolation clusters Phys. Rep. 54 1-74

[41] Morris J R and Coutts T J 1977 Electrical conduction in discontinuous metal films: a discussion Thin Solid Films $473-65$
[42] Dobierzewska-Mozrzymas E, Pieciul E, Bieganski P and Szymczak G 2001 Conduction mechanisms in discontinuous Pt films Cryst. Res. Technol. 36 1137-44

[43] Biegański P, Dobierzewska-Mozrzymas E, Pieciul E and Szymczak G 2004 Influence of micrsostructure on the conduction mechanism in discontinuous metal films on dielectric substrates Vacuum 74 211-6

[44] LeMieux M C, McConney M E, Lin Y-H, Singamaneni S, Jiang H, Bunning T J and Tsukruk V V 2006 Polymeric nanolayers as actuators for ultrasensitive thermal bimorphs Nano Lett. 6 730-4

[45] Raiteria R, Grattarola M, Butt H-J and Skládal P 2001 Micromechanical cantilever-based biosensors Sensors Actuators B 79 115-26

[46] Shipway A N, Katz E and Willner I 2000 Nanoparticle arrays on surfaces for electronics, optical, and sensor applications Chem. Phys. Chem. 1 18-52 